九州大学学術情報リポジトリ

Kyushu University Institutional Repository

\title{
A Note on Biometrics-based Authentication with Portable Device
}

Ohtsuka, Shinsuke

Faculty of Information Science and Electrical Engineering, Kyushu University

Kawamoto, Satoshi

Faculty of Information Science and Electrical Engineering, Kyushu University

Takano, Shigeru

Faculty of Information Science and Electrical Engineering, Kyushu University

Baba, Kensuke

Faculty of Information Science and Electrical Engineering, Kyushu University

他

http://hdl. handle. net/2324/15563

出版情報: Proceedings of the International Conference on Security and Cryptography. 2008, pp.99-102, 2008-06. INSTICC Press

バージョン :

権利関係 : 


\title{
A Note on Biometrics-based Authentication with Portable Device*
}

\author{
Shinsuke Ohtsuka Satoshi Kawamoto Shigeru Takano Kensuke Baba ${ }^{\dagger}$ \\ Hiroto Yasuura
}

\begin{abstract}
Individual authentication technologies are essential for electronic systems as social infrastructures. Especially, biometrics-based authentication has been receiving increasing attention and is expected to be implemented on systems with portable devices such as mobile phones for realizing more useful services. The most important problem in biometrics-based authentication is to prevent a leakage of biological information. This paper focuses on the leakage which enables a spoofing and consider two cases, a leakage from data stored in a server for verification of biological information and a leakage by a cheating detection. This paper proposes a solution by applying a function to biological information and shows the properties required for the function to solve the problem. Moreover, this paper proposes an idea of biometrics-based authentication system with portable devices which is provided a function to capture biological information.

keywords: Biometrics, authentication, mobile system, spoofing.
\end{abstract}

\section{Introduction}

Personal authentication has been one of the most important and fundamental techniques in our life as personal identification has become more common because of the requirement of certification of ATM cards, management of entering and leaving room or buildings, airport security system, and so on. Especially, as one of the techniques for identifying a person, biometricsbased authentication has attracted attention among many researchers in cryptography and computer security. This technique identifies a person by analysis of his/her physical characteristics which are called "biometric information" and is generally considered to be able to develop robust system against counterfeit attack thanks to their uniqueness and permanence. Furthermore, by introducing biometric authentication to a system based on mobile terminal devices and the

\footnotetext{
*An edited version of this report was published in: Proc. International Conference on Security and Cryptography (SECRYPT 2008), pp.99-102, INSTICC Press, Jul, 2008.

${ }^{\dagger}$ Faculty of Information Science and Electrical Engineering, Kyushu University, baba@i.kyushu-u.ac.jp
}

internet, more useful and effective services can be rendered to the users for mobile communications.

One of the most important problem in biometric authentication is to prevent the leakage of biometric information. To solve this problem, we have to pay attention to privacy concerns. Moreover, we need to be concerned that it is possible to create a counterfeit of a part of a living body [3]. In a certain authentication, a "prover" is the entity which is to prove that $\mathrm{s} / \mathrm{he}$ is a particular user and a "verifier" is the entity which is to verify the proof. Here we assume that secure communications including destination certifications are available by proper cryptographic technologies. And then, we do not consider the leakage of biometric information in daily life which is not concerned with procedure for personal authentication. And yet, under the above assumptions, it is conceivable that we can easily produce the leakage of biological information by guessing from verifier's information or biometric observation by fake verifier.

We propose the following method for solving the above problems. First, about the leakage of biometric information by guessing from verifier's information, we can solve it by applying the idea of "cancellable biometrics [4]". Now, we assume the case that the prover registers biometric information, which is observed in advance, as a "template" to the verifier. The main idea of cancelable biometrics is to apply a transformation, which is difficult to reconstruct the original information, to the observed biometric information so that the template can be cancelled. In short, by the transformation, the original biometric information remains secure even if there is a leakage of the template by security attacks. However, by pretending to be the verifier and observing the living body directly, one can obtain the original biometric information before the transformation is applied. This problem can be solved by using a mobile terminal with an ability for the biometric observation. If the mobile terminal is managed by a prover and the malware threat can be prevented, by applying an irreversible transformation to the observed biometric information on this terminal, one cannot reconstruct the original information by using every output of the terminal.

Firstly, in this paper, we propose a model of biometric-based authentication for the purpose of clar- 
ifying the above problems. In our proposed model, to consider the possibility of an unjust observation of biometric information, "scanner" is expressly formulated as an entity collecting biometric information by analyzing human body. To prevent the leakage of biometric information from the verifier's information, we have some requirements for the transformation which is applied to the biometric information. These requirements are corresponding to the property of the transformation to realize cancelable biometrics in the paper [4]. By applying such a transformation to the biometric information under the management of the prover, the effect is useful clearly for an unjust biometric observation.

This paper shows clearly that our approach can prevent the leakage of biometric information by using cancelable biometrics. Then, applying our method to a biometric-based authentication based on mobile terminal, we also illustrate to have the effect to prevent another possible type of leakage. Finally, we discuss the implementation about our method and present the problems of the biometric-based authentication using mobile terminal.

\section{Modeling Biometrics-based Authentication}

In this section, a model of biometrics-based authentication is introduced to bring out the problem we tackle in this paper. $\Sigma$ and $N$ denote the alphabet and the set of nonnegative integers, respectively.

\section{$2.1 \quad$ A Model}

In this paper, we consider identification of a user as authentication. Each of the users who can be a target of authentication is denoted by $u_{1}, u_{2}, \ldots \in U$. In a trial of authentication, a prover is the entity which is to prove that the prover is a particular user and a verifier is the entity which is to verify the proof. We consider a model with a single verifier in the rest of this paper. The atomic procedure of authentication is that the prover submits a string $w$ to the verifier, and the verifier decides who the prover is in $U$.

A key feature of our model is that biological information as digital sequences is distinguished from the living body of a target user, which enables us to examine leakage of biological information from a cheating scanner which detects biological information. The following argument does not depend on a kind of the part of a living body for biometrics-based authentication, therefore we regard a living body as the user who has it. A piece of biological information is a string over $\Sigma$ and the set of the pieces of biological information of $u_{i} \in U$ is $B_{i} \subset \Sigma^{*}$. For $R \subseteq N$, a scanner is a function $f: U \times R \rightarrow \Sigma^{*}$ which outputs a piece of biological information from a living body and a variable. Intuitively, this is modeling the situation that several kinds of biological information (in a sense of digital sequences) can be detected from a single living body. Then, the protocol of authentication is the following.

Protocol 1 (1) The prover puts $u \in U$ on the scanner;

(2) the scanner computes $f(u, r)$ for an $r \in R$;

(3) the scanner sends $f(u, r)$ as $w$ to the verifier;

(4) the verifier regards the prover as $u_{i} \in U$ if and only if $w \in B_{i}$.

In the previous protocol we are considering identification with no "ID", that is, the verifier does not know who the prover is (or insists) at the step (4). In the case where the prover sends his ID first, $u$ from the step (1) to (3) are replaced to $u_{i}$.

Now, we ignore a decline of an accuracy of authentication which is caused by the obscurity of biological information.

Assumption 1 For any $1 \leq i, j \leq|U|, B_{i}=$ $\left\{f\left(u_{i}, r\right) \mid r \in R\right\}$ and $B_{i} \cap B_{j}=\emptyset$ if $i \neq j$.

In practical systems of biometrics-based authentication, biological information as purely scanned data is usually large, and hence it is not practical that the verifier holds the $B_{i}$ to examine whether $w \in B_{i}$. The straightforward method to solve this problem is considering a function $g$ which is defined by an idea of a distance on strings and a threshold $c$ with respect to a string $t_{i} . t_{i}$ is called a template of $u_{i}$. Now, we ignore a decline of an accuracy of authentication which is caused by definition of a template and a distance on strings.

Assumption 2 There exist $g: \Sigma^{*} \times \Sigma^{*} \rightarrow N$ and $c \in N$ such that $\left\{b \mid g\left(t_{i}, b\right) \leq c, b \in \Sigma^{*}\right\}=B_{i}$ for any $1 \leq i \leq|U|$.

\subsection{Problems}

The problem we consider is a spoofing which is caused by leaked biological information. In fact, in some practical systems, it is possible to make a fake or artificial living-body from a piece of biological information [3]. Therefore, we assume the following in terms of the model introduced in the previous subsection.

Assumption 3 For any $1 \leq i \leq|U|$, a single $b \in B_{i}$ enable to make $u$ such that $f(u, r) \in B_{i}$ for $r \in R$.

Some cases of leakage of biological information caused by man-made factor (such as, carelessness of a verifier or a cheating verifier) are out of the scope of cryptographic technologies. On the assumption of the secure path by suitable cryptographic technologies, we focus on the following cases of the leakage: 
- leakage of a template at the verifier,

- leakage of a piece of biological information at the scanner.

In usual systems, a template is obtained by a reasonable feature-extraction based on biology from scanned biological information or is exactly the information. In this situation, biological information can be estimated from a leaked template and it enables a spoofing as the user of the template.

On our model, the naive method to decide a template is expressed by the condition that any element in $B_{i}$ can be $t_{i}$. Moreover, a straightforward featureextraction enables an estimation of the definition of the distance, that is, we should assume $g$ to be open. Therefore, the essential point of the former case of the leakage is that an element of $B_{i}$ can be estimated from $t_{i}$ by Assumption 2 even if $B_{i}$ cannot be obtained exactly. The latter case is exactly the leakage of $b \in B_{i}$. Thus, by Assumption 3 these cases enable the spoofing.

\section{Solutions}

To solve the problems in the previous section, we propose solutions by modifying biological information. The modification is expressed on the proposed model as a function from a string to a string with some properties. Moreover, we consider the entity which should apply the function to biological information.

\subsection{Leakage of Template at Verifier}

The problem of a spoofing by a leakage of a template from the verifier is expressed on the proposed model as that an element of $B_{i}$ can be estimated from $t_{i}$. In conclusion, this problem is solved by applying a generalized idea of "cancelable biometrics [4]", although the original idea is proposed to enable changing a template rather than to prevent a spoofing by a template. In fact, the results of this subsection are obtained by interpreting the argument in [4] into our model.

We consider to prevent a spoofing using $t_{i}$ by applying a function $\phi: \Sigma^{*} \rightarrow \Sigma^{*}$ to biological information. Let $t_{i}=\phi(b)$ for a $b \in B_{i}$. The prover (who has a living body) $u_{i}$ submits $\phi\left(b^{\prime}\right)$ for $b^{\prime} \in B_{i}$ as $w$ to the verifier. Then, on Assumption 1 and 2, the condition for realizing identification is described as the following property of $\phi$.

Condition 1 There exists $g^{\prime}$ such that, for any $p, q \in$ $\Sigma^{*}, g^{\prime}(\phi(p), \phi(q)) \leq c$ if and only if $g(p, q) \leq c$.

If we consider to add a step for applying $\phi$ into Protocol 1 , the possibility is only between the step (2) and
(3). Therefore, we assume that the scanner has a suitable function for it, that is, the scanner is redefined to be another function $f \circ \phi$ and whether $f(u, r) \in B_{i}$ is examined by $\phi(f(u, r))$ on the previous condition.

Protocol 2 (1) The prover puts $u \in U$ on the scanner;

(2) the scanner computes $\phi(f(u, r))$ for an $r \in R$;

(3) the scanner sends $\phi(f(u, r))$ as $w$ to the verifier;

(4) the verifier regards the prover as $u_{i} \in U$ if and only if $\phi^{-1}(w) \in B_{i}$.

Now, we do not assume any confidentiality of $g^{\prime}$ for preventing the spoofing. Then, a spoofing using $t_{i}$ can be prevented if $\phi$ has the following property.

Condition 2 For any $p \in \Sigma^{*}$, it is difficult to find $q$ such that $p=\phi(q)$ for $p$.

On the previous condition, the verifier does not always have the result of $\phi^{-1}(w)$ at the step (4) in Protocol 2. Formally, we have to refer the idea of "computational indistinguishability [2]" for the definition of the word "difficult". However, in some practical systems the properties of Condition 1 and 2 are not required strictly. The former guarantees the property of a kind of "collision-free" and the latter is the property of "one-way". On Condition 1, if a simple idea of distance is used as $g^{\prime}$, then an attack based on "hillclimbing" successes. Namely, in a search of $q$ such that $p=\phi(q)$ for a given $p \in \Sigma^{*}$, it is possible to have an $r$ such that $g(q, r)<g\left(q, r^{\prime}\right)$ by considering whether $g^{\prime}(\phi(q), \phi(r))<g^{\prime}\left(\phi(q), \phi\left(r^{\prime}\right)\right)$ recursively. This situation contradicts to Condition 2 in a strict sense. One of the solutions for this problem is to use a complex function as $g^{\prime}$. To find a suitable $\phi$ with $g^{\prime}$ is one of the difficulties for realizing a practical system based on the idea of cancelable biometrics.

\subsection{Leakage of Biological Information at Scanner}

As mentioned in Subsection 2.2, the same problem as the case of a leakage of a template is caused by a leakage of a piece of biological information at the scanner. Applying a function which has the properties of Condition 1 and 2 can prevent a spoofing using a template. However, if we consider a leakage of a piece of biological information at the scanner, we can not have the effect of this solution in systems of a naive implementation of this idea. We have to analyze the protocol from the viewpoint of the entity which should apply the function to biological information.

In practical systems with biometrics-based authentication such as a door access control system or an ATM, the scanner is usually managed by the prover as a part of the system. The prover cannot avoid a risk of the leakage of his biological information as long 
as he has to put his living body on a scanner which is not trustworthy.

A simple solution is that the prover manages the scanner and the function. In this case, the prover outputs only the result of $\phi(f(u, r))$ and hence $f(u, r)$ cannot be obtained from it by Condition 2. Therefore, a system based on this idea can prevent a leakage of $b \in B_{i}$ which enables a spoofing as $u_{i}$. A difficulty of this solution is how to implement a system with this idea. It is natural to consider a PDA or a mobile phone as the scanner which is managed by the prover since the devices have suitable functions for the computation of the functions, the scan of biological information, and the communication with other entity. Thus, it is useful in preventing spoofing in biometrics-based authentication systems to implement the functions to scan some kinds of living bodies on portable devices besides a camera and a microphone.

The assumption that the prover manages $f$ and $\phi$ yields another problem by "duplicated packet" or a kind of "replay attack". Namely, in the step (3) in Protocol 2 , the private scanner can send an old $\phi(f(u, r))$ as $w$. A simple solution for this problem is that the verifier adds $w$ which was accepted once into a negative list for the examination of $w \in B_{i}$. The essential solution is to recognize information which is intrinsic to living bodies, which is realized by using a special part of biological information such as information of a reflex action or applying the idea of "zero-knowledge [2]" into the detection at a scanner. To realize a function to recognize living bodies is one of the most important problems for biometrics-based authentication. Besides to find a part of a living body which contains information to enable the recognition of living body, it is also useful to apply the idea of "challenge and response [1]" into scanners on portable devices, for example, a camera with a special kind of flash. Note that this realizes authentication by the verifier of the prover instead of his portable device.

\section{Conclusions and Future Work}

We introduced a model of biometrics-based authentication and made the problem of spoofing by using leaked biological information clear. We proposed a solution to apply a function to biological information and showed the properties required for the function to solve the problem. Moreover, we proposed an idea of biometrics-based authentication system with a mobile device which has a function to detect biological information.

By the analysis of an implementation of the system, we can extract the following results: biometrics-based authentication which is secure against a spoofing can be realized by applying the idea of cancelable biomet- rics into a system with portable devices; and therefore, it is meaningful to implement functions to capture biological information on portable devices.

\section{References}

[1] H. Delfs and H. Kneble. Introduction to Cryptography - Principles and Applications. Springer, 2002.

[2] O. Goldreich. Foundation of Cryptography - Basic Tools. Cambridge University Press, 2001.

[3] T. Matsumoto, H. Matsumoto, K. Yamada, and S. Hoshino. Impact of artificial "gummy" fingers on fingerprint systems. In Proc. SPIE, Optical Security and Counterfeit Deterrence Techniques IV, volume 4677, pages 275-289, 2002.

[4] N. K. Ratha, J. H. Connell, and R. M. Bolle. Enhancing security and privacy in biometricsbased authentication system. IBM System Journal, 40(3), 2001. 